\title{
Technologies
}

\section{Le peloton cycliste}

De la culture technique à la sous-culture sportive

The Cyclist Bunch. From technical culture to sports subculture

\section{Xavier Garnotel}

\section{CpenEdition}

\section{Journals}

Édition électronique

URL : https://journals.openedition.org/tc/4904

DOI : $10.4000 /$ tc. 4904

ISSN : 1952-420X

Éditeur

Éditions de l'EHESS

\section{Édition imprimée}

Date de publication : 1 décembre 2009

Pagination : 306-329

ISBN : 978-2-7351-1301-9

ISSN : 0248-6016

Référence électronique

Xavier Garnotel, « Le peloton cycliste », Techniques \& Culture [En ligne], 52-53 | 2009, mis en ligne le 01 août 2012, consulté le 29 septembre 2022. URL : http://journals.openedition.org/tc/4904 ; DOI : https://doi.org/10.4000/tc.4904

Ce document a été généré automatiquement le 29 septembre 2022.

Tous droits réservés 


\section{Le peloton cycliste}

De la culture technique à la sous-culture sportive

The Cyclist Bunch. From technical culture to sports subculture

Xavier Garnotel

\section{NOTE DE L'ÉDITEUR}

Le corps du cycliste, façonné et sculpté, est l'instrument malléable des normes techniques du peloton. Il se présente également comme l'incarnation, le moteur dynamique d'une culture technique en évolution continuelle.

\section{NOTE DE L'AUTEUR}

Je tiens à remercier Mme Jeanjean et M. Biache pour leurs conseils avisés. 


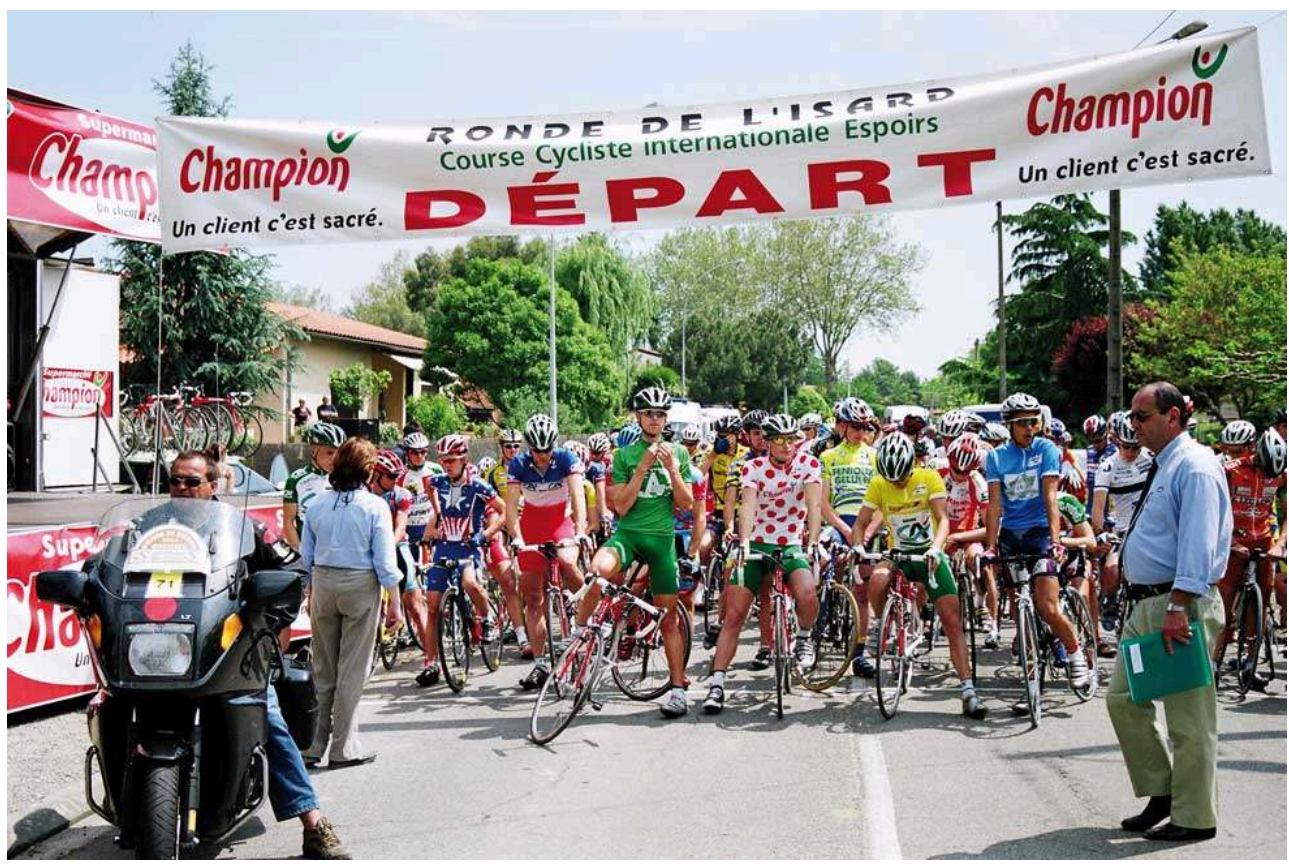

(c) X. Garnotel

L'initiation d'un jeune cycliste aux coutumes du peloton se caractérise par des traitements corporels spécifiques, comme le rasage des jambes, par «l'affûtage» de la musculature ou encore par l'accoutumance à une souffrance liée à la longueur des épreuves et à l'intensité des efforts. Ces techniques corporelles sont codifiées depuis la fin du XIXe siècle, ce dont témoignent les archives photographiques et journalistiques de cette époque. Plus qu'un agrégat de compétiteurs ou qu'un rassemblement de rivaux, le peloton regroupe des individus qui partagent la même façon de vivre et qui respectent également des codes sociaux transmis par une tradition orale.

1 La compréhension de ces pratiques et de ces représentations propres au peloton nécessite une méthodologie compréhensive, car l'appropriation et les reconstructions culturelles de l'activité par les pratiquants sont parfois en décalage avec les règlements officiels. Ainsi les outils de l'ethnologie apparaissent-ils pertinents pour appréhender ces phénomènes sociaux. J'ai intégré un peloton cycliste amateur en participant aux épreuves afin d'observer les pratiques des coureurs in situ, pendant les courses, mais aussi au sein de l'équipe en partageant son quotidien ${ }^{1}$.

2 La démarche inductive de mon enquête de terrain m'a amené à constater que la plupart des théorisations classiques des cultures sportives semblaient inopérantes et incapables d'expliquer ce qui se joue véritablement au sein du peloton. L'interprétation théorique a donc été formulée à la suite de l'observation participante, à partir d'un travail comparatif avec d'autres études portant sur les pratiques corporelles.

3 Cet article a pour but de comprendre ce qui caractérise l'existence d'un cycliste et d'analyser les mécanismes de construction de cette pratique. La problématique consiste à étudier comment les pratiquants l'ont élaborée, en s'adaptant pragmatiquement aux "propriétés ${ }^{2}$ techniques de l'engin et aux propriétés physiques des épreuves. Il apparaît dès lors que « vivre cycliste » nécessite une ascèse normée par des techniques corporelles inscrites dans une temporalité précise et soumise à une organisation sociale. Ce façonnage technique s'accompagne de jugements de valeur sur l'esthétique du mouvement et du corps. Les marqueurs corporels de cette esthétique sont liés à leur 
aspect fonctionnel et à leur efficacité. Ils ont ainsi pris une dimension stylistique qui doit être mise en relation avec le système hiérarchique du peloton.

\section{Les propriétés physiques, techniques et réglementaires du sport cycliste}

\section{Les propriétés techniques de l'engin}

Les structures techniques de la bicyclette favorisent le rendement des déplacements. La bicyclette actuelle n'est pas un simple outil qui ne ferait qu'augmenter une fonction anatomique humaine. Elle n'est pas non plus une machine qui permettrait d'accomplir des actions autonomes sans effort ni volonté constante de la part de son utilisateur. Elle augmente donc le rendement de l'effort mais ne s'y substitue pas. Les propriétés physiques et réglementaires des courses cyclistes découlent des caractéristiques techniques de cet engin. Il aurait été en effet impossible de proposer des épreuves de plusieurs centaines de kilomètres avec un engin comme la draisienne, une "machine à rouler » inventée en 1818, qui permettait à son utilisateur de pousser avec ses jambes et de se laisser rouler par le système basique de la roue, connu depuis l'Antiquité. En revanche, avec l'invention des pédales par les frères Michaux en 1861, le système de transmission par le pédalier permit d'augmenter le rendement de la force motrice. Cette innovation induisit rapidement une adaptation technique. En effet, le pédalier, fixé sur la roue avant permettait de réaliser un déplacement égal à la circonférence de la roue avant à chaque tour de pédale. La démarche des industriels consista alors à augmenter la circonférence de la roue avant pour accroître la distance parcourue à chaque tour de pédale par le vélocipédiste : ce fut l'essor des grand-bi qui fleurirent à la fin $d u X x^{e}$ siècle. Cet engin, malgré son instabilité, due à sa hauteur, augmentait le rendement et la vitesse du déplacement. Ainsi la première course de vitesse officielle eut lieu en mai 1868 sur un circuit de 1200 mètres à Saint-Cloud dans les Hauts-deSeine. Puis, les sciences et les procédés techniques de l'ère industrielle permirent de réaliser la chaîne articulée grâce aux technologies de l'acier (Gille 1966). La transmission du mouvement par la chaîne a rendu motrice la roue arrière (Vigarello 1988). Elle a également permis la mise en place de la démultiplication de la force par le choix du braquet. Parés de la transmission puis de la démultiplication de la force, les engins possédaient une fiabilité et un rendement supérieurs. Le grand-bi a alors disparu au profit des bicyclettes «traditionnelles », ayant deux roues d'égal diamètre.

4 Les structures techniques du vélo de course étaient alors définies et réglementées par des textes institutionnels. En effet, depuis leur création, l'Union vélocipédique de France, fondée en 1881 (qui deviendra la Fédération française de cyclisme en 1941), et l'Union cycliste internationale (U.C.I. fondée le 14 avril 1900) régulent les innovations techniques pour conserver la valeur essentielle du « cyclisme traditionnel » : conserver le primat de l'homme sur la machine.

5 La bicyclette possède dès lors des propriétés de rendement et de vitesse par le roulement, la démultiplication de la force motrice et l'ensemble des autres éléments techniques qui contribuent à son confort et à sa stabilité (le système de freinage, les pneumatiques, puis le dérailleur permettant de changer de développement...). Les organisateurs et les coureurs ont défié depuis la distance à travers de grands périples. Le premier Bordeaux-Paris fut couru le 23 mai 1891 sur une distance de $580 \mathrm{~km}$ à 
22,3 km/h, et du 6 au 9 septembre 1891, le célèbre Paris-Brest-Paris de 1200 kilomètres a été remporté par Charles Terront en plus de 71 heures (Paturle \& Rebière 1997).

\section{Le contexte socio-économique et les propriétés physiques des saisons cyclistes}

Les courses cyclistes constituent depuis leur origine des vitrines commerciales pour les industriels. Ainsi leur multiplication relève pour une grande part d'une volonté de promotion commerciale. Il s'agit en effet d'une surenchère d'exploits physiques qui a été orchestrée par des promoteurs sportifs dans le but de se faire connaître du grand public et de vendre leurs produits (journaux, matériels de cycles...). Par exemple, le Tour de France, la plus grande épreuve cycliste mondiale, est né d'une scission du journal Le Vélo et du journal L'Auto. Le Vélo était tiré à plus de 80000 exemplaires jusqu'en 1900. C'est alors qu'il prit position pour les dreyfusards, sous la plume de Pierre Giffard. Le comte de Dion décida alors de fonder un journal concurrent " apolitique » : L'Auto. La conjoncture se prêtait à l'instauration de ce deuxième journal spécialisé puisque la popularité des courses cyclistes était croissante. L'Auto prendra définitivement l'ascendant en lançant le Tour de France du 1er au 19 juillet 1903, lequel proposait alors un parcours de 2428 kilomètres en huit étapes (Gaboriau 1995). La quasi-totalité des grandes épreuves cyclistes internationales datent de cette époque : Liège-Bastogne-Liège "la doyenne » en 1892, Paris-Roubaix «l'enfer du Nord » en 1896, Milan-San Remo en 1907, le Tour d'Italie. « Giro » fut fondé, quant à lui, le 13 mai 1909 avec 2448 kilomètres en six étapes.

Ces courses, devenues des épreuves sportives internationales, se caractérisent toujours par leur longueur (entre 200 et 300 kilomètres parcourus à plus de $40 \mathrm{~km} / \mathrm{h}$ pour les épreuves professionnelles actuelles). Elles exigent une grande endurance ainsi qu'une importante résistance physique. La dramaturgie intrinsèque du spectacle mis en scène par les organisateurs repose sur les contraintes imposées aux «forçats de la route» (Londres 1924). 


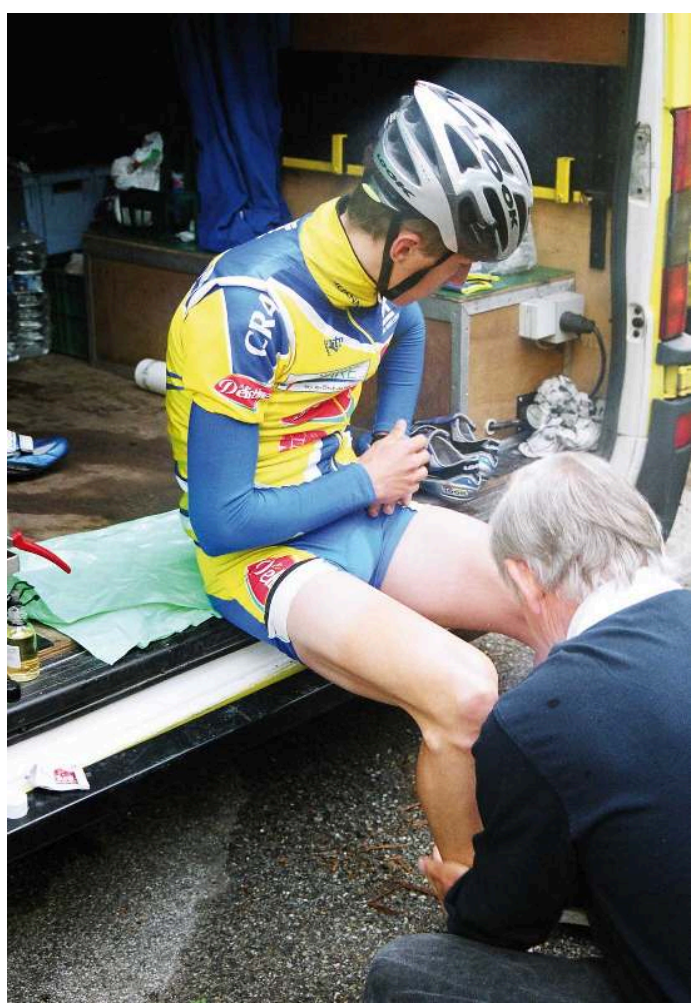

(c) X. Garnotel

2004, sur la Ronde de l'Izard, le masseur de l'équipe de Roanne enduit les jambes d'un coureur d'huile camphrée avant le départ d'une course pluvieuse.

Les propriétés de longueur et d'exigence physique des courses cyclistes constituent un construit technique, économique et social. En plus de la distance des épreuves, une saison cycliste se caractérise par la répétition des compétitions. Cela correspond également à une surenchère médiatique et organisationnelle de la part des promoteurs qui ont densifié le calendrier en multipliant les dates de compétitions. Cela est vrai à partir du niveau régional, où l'on retrouve plusieurs courses champêtres et villageoises chaque dimanche et pendant les jours fériés. Cette fréquence est également liée à un paramètre physique. En effet, même si la demande de spectacles était identique pour le marathon, par exemple, il serait physiquement impossible pour un athlète de cumuler plus de trois à cinq marathons dans l'année. Le contact avec le sol de la course à pied engendre des traumatismes tendino-articulaires qui nécessitent deux à trois mois de régénération et d'entraînement modéré. Ce facteur n'existe pas en cyclisme, lequel ne présente aucun risque de traumatisme de ce type, si l'on exclut les tendinites liées aux mauvais réglages de position sur l'engin et les blessures liées aux chutes. Il est ainsi physiquement possible d'accumuler un nombre très élevé de journées de courses, entre 120 et 160 à l'année pour les professionnels. Si cela ne provoque pas de traumatismes articulaires, cette cadence s'apparente parfois aux travaux forcés et épuise l'organisme qui peut tomber en syndrome de surentrainement ou d'épuisement chronique.

Le peloton, que nous entendons comme ce regroupement de compétiteurs et, plus encore, comme cette sous-culture sportive composée de tout l'encadrement des équipes qui partagent la même façon de vivre, a élaboré, depuis plus d'un siècle, une tradition technique pour encaisser ces charges de travail (qui représentent près de 20000 kilomètres pour les meilleurs amateurs et plus de 35000 à 40000 kilomètres parcourus à vélo, par an, pour les professionnels). 


\section{Les techniques corporelles du « métier»}

\section{Des techniques du corps qui façonnent l'organisme}

"C'est l'ensemble de toute une saison, en fin de compte si tu veux faire une bonne saison, faut partir sur de bonnes bases, et la base tu la fais l'hiver, donc en fait ce qui te pousse à y aller c'est que tu te projettes dans la saison. Tu te dis, si je veux que ça marche, c'est maintenant. C'est peut-être aussi une préparation mentale, braver le froid, la pluie, faire des intervalles, je sais pas si physiquement, enfin ça doit quand même t'apporter quelque chose, mais au niveau du mental tu te dis j'ai fait tout ce qu'y avait à faire, t'as pas de doute » (S.M., coureur Élite 2).

9 Un coureur cycliste s'emploie au quotidien à respecter les normes du« métier». Ce terme du jargon cycliste renvoie à la culture technique du peloton et à ses contraintes alimentaires, à ses exigences de repos et d'entraînement. Il s'agit d'une véritable ascèse rythmée par des techniques correspondant aux entraînements prescrits. Au-delà des heures de préparation physique, le corps est façonné par un ensemble de «techniques du corps » (Mauss 1968), destinées à accroître la récupération, afin d'augmenter la capacité à reproduire des charges d'entraînement et de compétition. Ces techniques du corps sont également codifiées temporellement. La préparation technique suit généralement un scénario cyclique qu'il faut respecter pour se présenter dans les meilleures conditions, selon la tradition du peloton. Cette codification des techniques du quotidien s'apparente ainsi à une sorte de "chaîne opératoire " (Leroi-Gourhan 1945) dont l'objet est le corps. Bromberger la définit comme une «syntaxe organisée d'actions, associant gestes, outils, connaissances, aboutissant à la transformation d'une matière première en un produit fabriqué » (1986).

Le coureur doit en effet mettre en correspondance le temps du corps, avec ses aléas, ses troubles physiques, son rythme propre et le temps social, celui du calendrier, des épreuves à répétition, et atteindre les grands objectifs où il faut essayer d'arriver au summum de ses capacités. Le cycliste s'inscrit dans une temporalité cyclique à différentes échelles. Celle des saisons avec une mise en action progressive lors de la période hivernale où il faut "manger de la borne » en réalisant une préparation foncière pour travailler l'endurance de base, en augmentant le rythme des séances à l'approche des compétitions. Ensuite, la saison sportive est rythmée par des cycles avec les classiques printanières, les tours estivaux pour se terminer par des critériums ${ }^{3}$. À une échelle temporelle plus courte, la semaine suit également un cycle composé de techniques qui permettent d'équilibrer le relâchement du tonus lors des «sorties de décontraction, de décrassage » et la mise en tension progressive afin de se présenter au mieux à la compétition, qui a le plus souvent lieu le week-end.

Ainsi, il existe une multitude de techniques de soin du corps, comme celle de se couvrir les jambes au repos. À l'entraînement, les coureurs ne se les découvrent que rarement avant les mois de mars, avril. Au-delà de l'aspect fonctionnel, le cuissard long ou les jambières (descendant jusqu'aux chevilles) représentent la protection, les coureurs les mettent en début de saison, la veille des courses, à l'échauffement avant de les enlever quelques minutes avant l'épreuve pour se mettre "en court». Le cuissard court, ce vêtement aérodynamique moulant les cuisses du coureur, constitue un élément caractéristique du statut de cycliste, son usage technique et -fonctionnel s'associant également à l'appropriation sociale et stylistique de cet objet. 

chaque course pluvieuse ou par temps froid, les techniciens des équipes ou les membres de la famille massent les coureurs avec des crèmes chauffantes et des huiles à base de camphre dont l'odeur donne un parfum caractéristique sur la ligne de départ. Ces onctions ont également lieu après les étapes avec des crèmes hydratantes, notamment au niveau de l'aine afin d'éviter les irritations dues aux frottements avec la selle, malgré la peau de chamois du cuissard (une protection cousue à l'intérieur du cuissard pour améliorer le confort du coureur).

13 Les cyclistes essayent de ménager leur corps à tous les instants de leur vie quotidienne par l'intermédiaire de techniques de repos et de sommeil : «Reste assis, faut que tu fasses $d u$ jus » dit un entraîneur d'un club amateur à l'un de "ses jeunes ». On observe que plus un coureur supporte de charges d'entraînement élevées, plus les techniques de repos et de sommeil deviennent importantes. Pour le cycliste, le repos et le sommeil renvoient à une activité $\mathrm{du}$ « métier » : la récupération. Les techniques de sommeil dans les déplacements des courses par étapes professionnelles font l'objet d'attentions particulières : il faut que les coureurs rentrent le plus vite possible à l'hôtel, pour se faire masser, se restaurer et se coucher tôt. Les cars sont aménagés pour permettre d'avoir les jambes surélevées et couvertes. L'ambiance dans les hôtels, lors des courses par étapes professionnelles et au bout de deux semaines, est d'un calme absolu. Les coureurs marchent doucement, sans brusquer leurs muscles, se protègent le corps contre le froid, procèdent à des soins corporels méticuleux. Certes, les coureurs transgressent parfois cette règle de vie. Certains récits ont romancé les victoires de grands champions aux lendemains de sorties nocturnes (à propos de Jacques Anquetil par exemple). Mais ces écarts sont suffisamment occasionnels pour être narrés dans la littérature et être racontés de façon facétieuse par les coureurs.

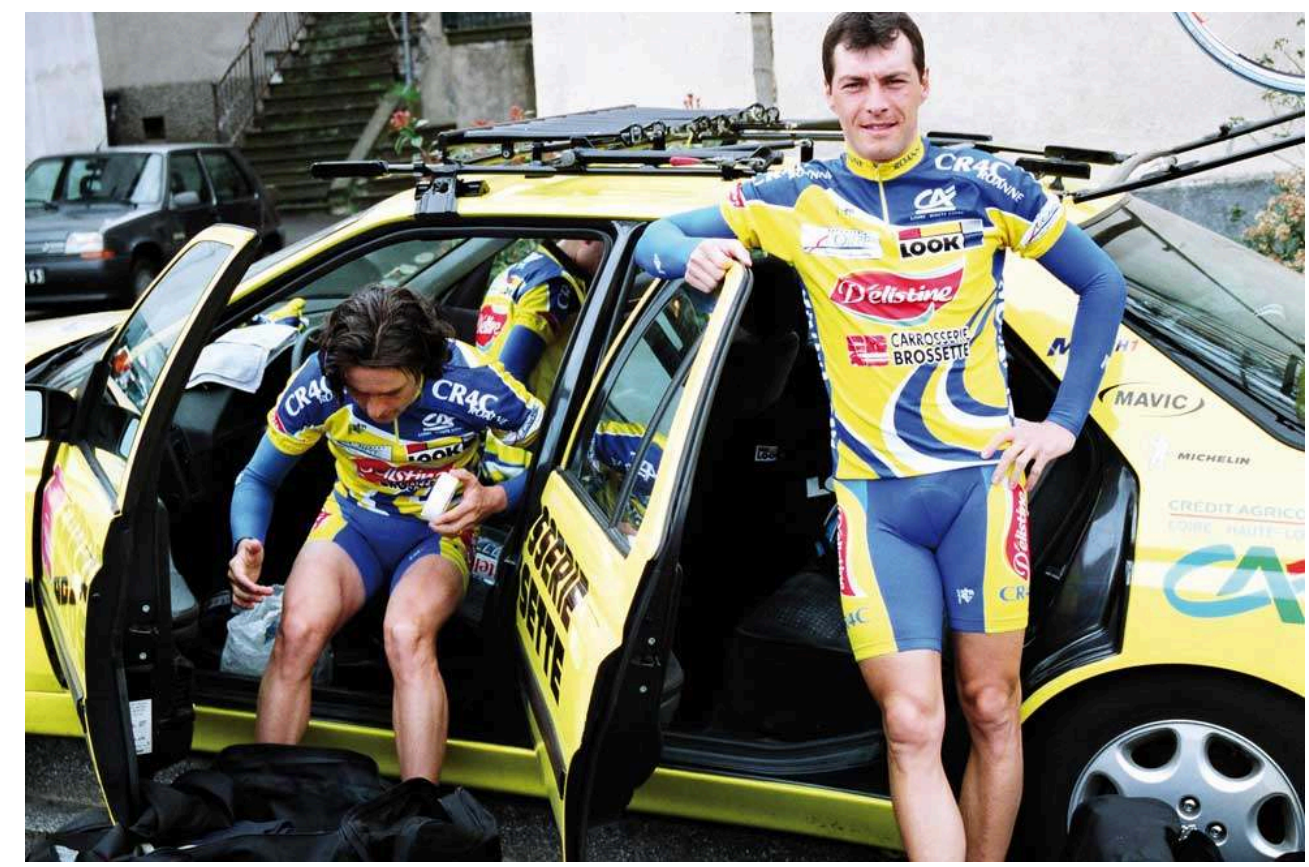

(c) X. Garnotel

Les dernières préparations corporelles avant le départ.

14 En effet, l'humour est très présent dans les équipes cyclistes. Il possède une fonction cathartique en permettant d'évacuer les tensions physiques et psychiques. La 
monotonie, la rigueur et la souffrance en sont ainsi adoucies et atténuées. De nombreuses enquêtes ethnologiques ont également identifié cette fonction cathartique de l'humour dans des sociétés où le quotidien est difficile et éprouvant. Le rire permet «à la fois [de] parler de choses graves et [de] faire rire ceux qui les écoutent » (Clastres 1974). L'ascèse facétieuse des cyclistes ironise ainsi sur les rapports hiérarchiques. Cela permet de mettre, en quelque sorte, de l'huile dans les rouages des équipes où les ambitions de pouvoir et de leadership sont omniprésentes. L'humour des cyclistes utilise abondamment les éléments naturels et du quotidien pour créer des moments de convivialité et de rire. Ainsi, l'hygiène des besoins naturels fait l'objet d'attentions particulières. La vie collective, et quasi exclusivement masculine, des équipes, rassemblées dans les hôtels ou dans des dortoirs collectifs tend à faire partager l'intimité de chacun et à utiliser avec abondance l'humour scatologique. Les différents états de la défécation et des flatulences apparaissent codifiés comme des «signes de forme» ou au contraire de déclin physique : "Ça c'est le chef, j'le reconnais à l'odeur ", "T'es pas trop en forme en ce moment ", "Salop, si tu marches pas avec ça!»

Les activités sexuelles sont également évoquées et soumises à la plaisanterie. Il est généralement conseillé de ne pas avoir de rapport sexuel à la veille d'une course car cela couperait "l'influx nerveux». À cet effet, il faut noter la séparation des sexes lors des courses par étapes isolant les coureurs de leur compagne. La coutume incite également à ne pas se raser et à ne pas se doucher le jour de l'épreuve, car une nouvelle fois, cela couperait «l'influx». Cette expression récurrente du jargon, notamment utilisée par les entrâneurs, est un terme scientifique (caractérisant la tonicité musculaire et son innervation) sorti de son contexte. (C'est le cas d'autres notions comme par exemple celle d'« acide lactique »).

\section{Le corps et ses croyances}

Devant la longueur des saisons et l'incertitude qui demeure autour de chaque préparation physique, le corps préserve son mystère et laisse parfois place à de soudaines défaillances. Cela fait partie de l'expérience du cycliste qui tente alors de modifier ses entraînements et d'adapter son rapport au temps.

«En fait, on est toujours sur une corde raide, y suffit qu'y ait un petit truc qui te dérègle et tu t'enfonces de plus en plus. Ça fait depuis le Béda que je sens que ça va pas trop, et j'ai insisté, au Val d'Allier, j'me suis dépouillé pour faire quatrième et là je sens bien que suis complètement sec. Je sens comme des pics dans les jambes aux moments où ça embraye, j'ai mal au dos et j'ai plus le goût. Pourtant j'ai fait une prise de sang et les docteurs ont rien trouvé, j'ai jamais eu d'aussi bonnes analyses. Donc j'vais couper, faire de la promenade, pour revenir plus tard et on verra » (S.M.). 


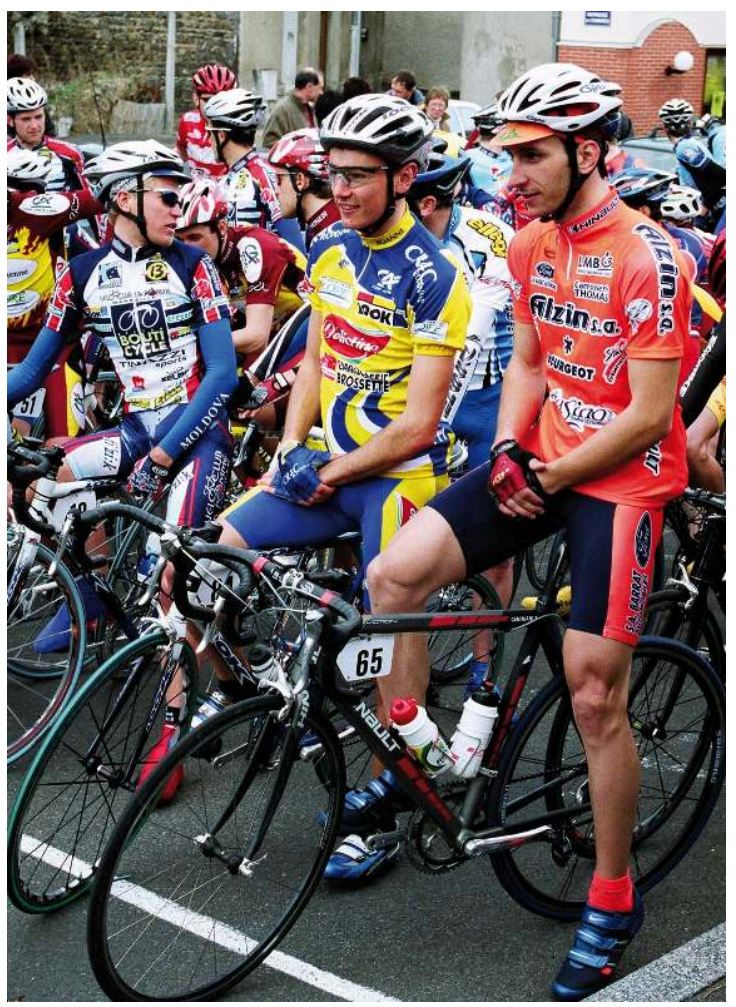

(c) X. Garnotel

L'ambiance sur la ligne de départ : Odeur de camphre, discussions et facéties pour évacuer la tension et l'enjeu.

Certaines pratiques spécifiques au peloton et certaines sensations corporelles se justifient donc à travers des termes issus des sciences. Ils sont intégrés dans le jargon, aux côtés d'expressions imagées et métaphoriques comme "des pics dans les jambes » pour la douleur provoquée par l'acide lactique, des « socquettes en titane ", caractérisant la bonne forme physique ou à l'inverse des "jambes en guimauve ", illustrant le peu de dynamisme du moment.

Ces termes se distinguent donc des croyances clairement empiriques qui sont transmises par la tradition orale du peloton, sans aucun recours aux sciences. Il s'agit là de "la vieille école », des "savoirs des anciens» qui sont toujours à l'œuvre au sein du peloton et que les termes mentionnés du jargon illustrent. Mais les discours apparemment scientifiques des préparateurs et des "coachs" modernes ne le sont que rarement de part en part. Tout comme dans les discours des entraîneurs et des enseignants de gymnastique, il apparaît que

«Théorie et empirie se croisent mutuellement dans un processus réciproque de renforcement de la croyance. Ce renforcement consiste, d'une part, en un "remplissement" empirique de l'argument théorique permettant de le déterminer (non pas conceptuellement, mais métaphoriquement le plus souvent), et d'autre part, en une caution intellectuellement élaborée, allusivement scientifique et socialement finalisée, fournie à la proposition empirique. Ce processus de renforcement réciproque permet de mieux comprendre comment un ensemble de croyances peut prendre une certaine consistance, s'indurer en tant que système " (Cizeron 2009: 166).

Il existe ainsi au sein du peloton des interdictions alimentaires et sexuelles, des traitements du corps et de la pilosité que l'on retrouve dans un grand nombre de 
sociétés avant une épreuve physique ou un rite de passage. Segalen a d'ailleurs illustré la dimension rituelle des pratiques corporelles contemporaines en caractérisant leurs rapports particuliers au corps, à autrui et à l'espace-temps (1998). Les pratiques du peloton fonctionnent et se transmettent de façon traditionnelle. La légitimation s'effectue sur le registre de la croyance par l'intermédiaire de certitudes affirmées par les entraîneurs et les familles. Au-delà des bienfaits physiologiques de ces techniques du corps, la croyance en leur efficacité « donne de la confiance » au coureur. Les mystères du corps et de la forme laissent place à des habitudes associées à un système de représentation qui intègre les techniques dans une temporalité précise.

\section{Des techniques intégrées dans une organisation coutumière}

En ce qui concerne les techniques de consommation alimentaire, on s'aperçoit que la dimension gustative est écartée au profit de la dimension fonctionnelle. Le respect de ces préceptes techniques est évidemment plus ou moins assidu selon le niveau de pratique. En principe, à la veille de la course, un coureur absorbe des quantités très importantes de pâtes : 200 grammes la veille au soir : «c'est le carburant ». Le jour de la course, il faut prendre un petit-déjeuner riche en sucres lents et un repas très léger, trois heures avant la course. En revanche, les viandes rouges sont déconseillées car elles présenteraient «des toxines » et les cyclistes n'absorbent que des viandes blanches lors des deux ou trois repas précédant la course.

Malgré toutes ces attentions portées au corps, les charges d'entraînement sont colossales. Cette pratique est " anormale » sur le plan physiologique et l'organisme ne peut se régénérer avec une alimentation usuelle. Ainsi, l'utilisation de compléments alimentaires sous formes de gélules et même de piqûres intramusculaires au niveau des muscles fessiers (notamment pour les carences en fer) est fréquente, même chez les juniors (âgés de 17 à 18 ans). Ces prises de cachets ou de "fléchettes» s'intègrent dans les us des pratiquants. Il faut alors dissocier la prise occasionnelle d'adjuvants du dopage organisé. La première forme que le jargon nomme «bricolage» utilise principalement des produits comme la cortisone, les anabolisants ou les euphorisants. Il s'agit principalement de coureurs amateurs qui «chargent la mule » en connaissant plus ou moins bien les subtilités de la posologie de ces remèdes. La seconde nécessite, quant à elle, des moyens et une organisation importants. Les techniques corporelles quotidiennes sont alors associées aux prises de ces produits interdits par les règlements :

" "J" moins trois semaines : toujours mes injections de fer, de B12, de Prefolic et d'EPO, ces dernières en sous-cutané dans le ventre, avec les mêmes fréquences. Je commençais en outre ma cure d'hormones de croissance, deux unités quotidiennes sur huit jours injectées en intramusculaire » (Chiotti 2001).

De nombreuses autres révélations éditées décrivent les prises de différentes substances telles le «pot belge » (un concentré de caféine, d'éphédrine, de cocaïne...) ou encore l'E.P.O. (Voet 1999). Ces témoignages dévoilent une organisation sociale de pratiques de dopage utilisant des biotechnologies (et donc moins accessibles aux instances de contrôles) présente dans certaines équipes ou groupuscules professionnels qui nécessite une logistique complexe et des soigneurs, qu'ils soient anciens coureurs, kinésithérapeutes ou médecins. De plus, ce dopage ajoute une dimension sociale à la technique de consommation : 
« Je me souviens exactement ce que j'ai ressenti juste après. C'est triste à dire, mais j'étais fier d'avoir fait cette piqûre. Je venais de franchir le pas, j'étais devenu un vrai pro, un homme. J'éprouvais vraiment de la satisfaction à avoir fait tomber cette barrière. Derrière, il y a une notion de courage. D'ailleurs, j'étais tout de suite allé voir quelques coureurs [...] pour leur dire: "Ça y est! J'ai fait mon premier Kenacort!" » (Gaumont 2005 : 108).

Cet extrait met en lumière l'aspect initiatique de l'absorption d'un produit interdit ou réservé aux malades (hormones de croissance, E.P.O...). Il donne à voir un mécanisme d'intégration au sein de ce que la sociologie catégorise justement comme une forme de société secrète. Pour le nouvel initié apparaît un sentiment de fierté, de passage vers un statut d'adulte et d'appartenance à un groupe d'élite. Il exprime le sentiment d'assumer ses responsabilités et d'exploiter ses dons naturels: "j'étais devenu un vrai pro, un homme ».

Il est évident que ces techniques de dopage s'inscrivent dans le contexte économique global des sports spectacles contemporains utilisant, au plus haut niveau mondial, les biotechnologies les plus récentes. Pourtant, il existe des particularités propres au milieu cycliste ${ }^{4}$. La prise d'adjuvants et de substances classées comme illicites remonte ainsi aux origines des compétitions cyclistes. En effet, les frères Pélissier en témoignent dès 1924 dans le célèbre article d'Albert Londres « Les Forçats de la route » :

«Voulez-vous voir comment nous marchons ? Tenez...

De son sac, il sort une fiole:

- Ça, c'est de la cocaïne pour les yeux, ça, c'est du chloroforme pour les gencives...

- Ça, dit Ville, vidant aussi sa musette, c'est de la pommade pour me chauffer les genoux.

- Et des pilules? Voulez-vous voir des pilules? Tenez, voilà des pilules

Ils en sortent trois boîtes chacun.

- Bref, dit Francis, nous marchons à la dynamite » (Londres 1924).

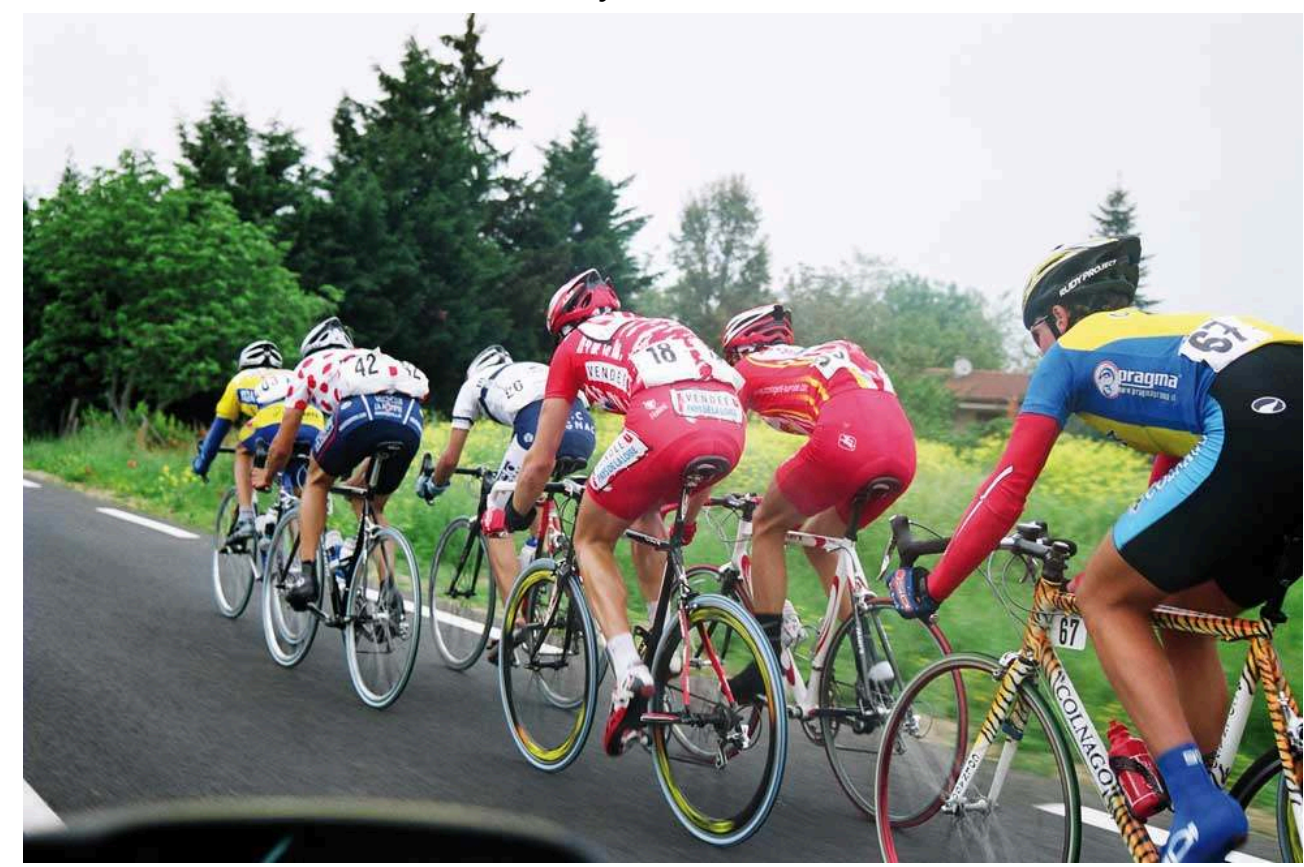

(c) X. Garnotel

Les coureurs d'une échappée « tournent » en se passant des relais.

Cette pratique de dopage, qui focalise les attentions journalistiques et populaires en suscitant des questions morales sur les notions d'équité ou de santé publique, s'intègre 
plus largement dans un ensemble technique et social au sein des équipes. Les techniques de dopage du peloton sont nécessairement liées aux propriétés d'un sport qui tend inexorablement à repousser les limites physiologiques du corps humain. En effet, il y a un «étiquetage » (au sens de Becker) arbitraire et institutionnel de certaines substances "dopantes", alors que le processus de consommation d'adjuvants suit une initiation au fur et à mesure de la «carrière » de cycliste : incorporation des normes techniques, optimisation des techniques d'entraînement et perfectionnement au sein de l'élite.

De plus, cette logique s'insère dans l'organisation coutumière du peloton, celle d'une régulation spontanée qui a construit des codes sociaux et des régulations économiques particulières. La construction de ces organisations sociales propres au peloton a été structurée par l'adaptation des pratiquants aux propriétés physiques et techniques de ce sport. En effet, le vélo démultiplie la force motrice et améliore le rendement. Ainsi, un cycliste se déplace plus rapidement et à moindre coût énergétique qu'un coureur à pied. La résistance à l'avancement est donc plus importante en cyclisme que dans les autres sports de courses libres (si ce n'est le roller de vitesse où l'on observe également des organisations collectives adaptées au vent et au phénomène d'aspiration puisque les patins à roulettes améliorent également le rendement et la vitesse de déplacement). La différence d'énergie dépensée entre un cycliste qui mène un groupe et ceux qui sont "abrités » (dans le sillage d'un concurrent) est très importante, environ $30 \%$ de différence à $40 \mathrm{~km} / \mathrm{h}$ (Monod \& Flandrois 2003).

Les coureurs ont alors construit des codes et des valeurs qui permettent de réguler ces temps de passage. Ils ont codifié des organisations hiérarchiques au sein des équipes pour protéger les leaders en faisant travailler les équipiers aux avants du peloton. Il se forme parfois même sur certains critériums cyclistes des «mafias", terme utilisé par le peloton pour caractériser des ententes illicites entre des coureurs qui contrôlent tactiquement la course pour faire gagner un coureur qui achète sa victoire. Cela fait partie des arrangements et de la réappropriation de la pratique par les coureurs, audelà des règlements officiels et des règles institutionnelles (Garnotel 2009b). Ces éléments techniques et sociaux sont en conflit avec les valeurs morales des institutions sportives. Elles sont ainsi considérées comme déviantes par rapport au modèle dominant. Il s'agit là de l'une des caractéristiques majeures de la notion de sousculture. Cette culture cycliste se caractérise également par des codes sociaux qui permettent aux membres d'une équipe de coopérer.

\section{Une organisation technique et sociale au sein des équipes}

L'organisation d'une équipe est sous la responsabilité du directeur sportif, un chef, généralement charismatique et expérimenté, fort d'une carrière reconnue par ses coureurs. Certains directeurs sportifs sont d'anciens grands professionnels, d'autres, dont la carrière fut plus modeste, font leur réputation progressivement en tant qu'accompagnateurs.

«On a aucun lien de parenté, on est simplement obligé de monter un groupe pour vivre dans les meilleures conditions, pendant des laps de temps longs, de février à octobre sur les différents circuits et les différentes courses par étapes. [...] Le directeur sportif a affaire à des personnes en difficulté, face à une certaine souffrance, en train d'encaisser du mauvais temps, du froid, de la pluie, nous, on est la béquille, la personne sécurisante. Le coureur il n'a plus personne, il a une maison, sur quatre roues qui suit la course, il faut qu'il ait tout son confort, même si c'est 
qu'une voiture. Faire de la place aux coureurs, leur donner la priorité. Quand on a un transfert, arrivée d'étape à l'hôtel, garer les véhicules ensemble, le plus près possible de la chambre d'hôtel pour éviter que le groupe fatigué se disperse » (Y.G., directeur sportif d'équipes de division nationale amateur).

Le directeur sportif encadre un groupe composé de plus de 35 personnes chez les professionnels. Il définit aussi les stratégies d'équipe, le leadership ${ }^{5}$, et veille au confort de ses protégés. Un coach ${ }^{6}$ a également la responsabilité de coordonner le travail des différents techniciens de l'équipe. Parmi eux, le masseur entretient un rapport particulier avec le coureur.

«La plupart des masseurs d'équipes sont d'anciens coureurs, et quand t'as eu de bonnes sensations de massage, t'as envie de les retransmettre en tout cas c'est pas évident. [...] C'est plus des sensations que l'on a ressenties comme coureur, le mal de jambes, moi j'ai l'impression d'avoir couru hier encore, le mal de jambes, il est dans ma tête pour la vie, incroyable. [...] Une course par étapes sans massage c'est impossible, vous allez tenir trois jours et après les muscles n'ont plus leurs qualités. Mais certaines fois on a l'impression qu'il y a une poignée de cailloux dans les muscles, c'est noué, c'est très difficile à sortir. »

$\mathrm{XG}:$ « À sortir, c'est sortir quoi ?»

$\mathrm{PL}:$ «À sortir les toxines. Tu sens vraiment des bosses dans le muscle. C'est très dur quand ils sont vraiment toxinés, surtout quand il a eu des étapes de montagne avec le froid, la pluie c'est sûr que c'est beaucoup plus dur, beaucoup plus fatigant qu'une journée au soleil » (P.L., masseur au sein de l'équipe professionnelle AG2R).

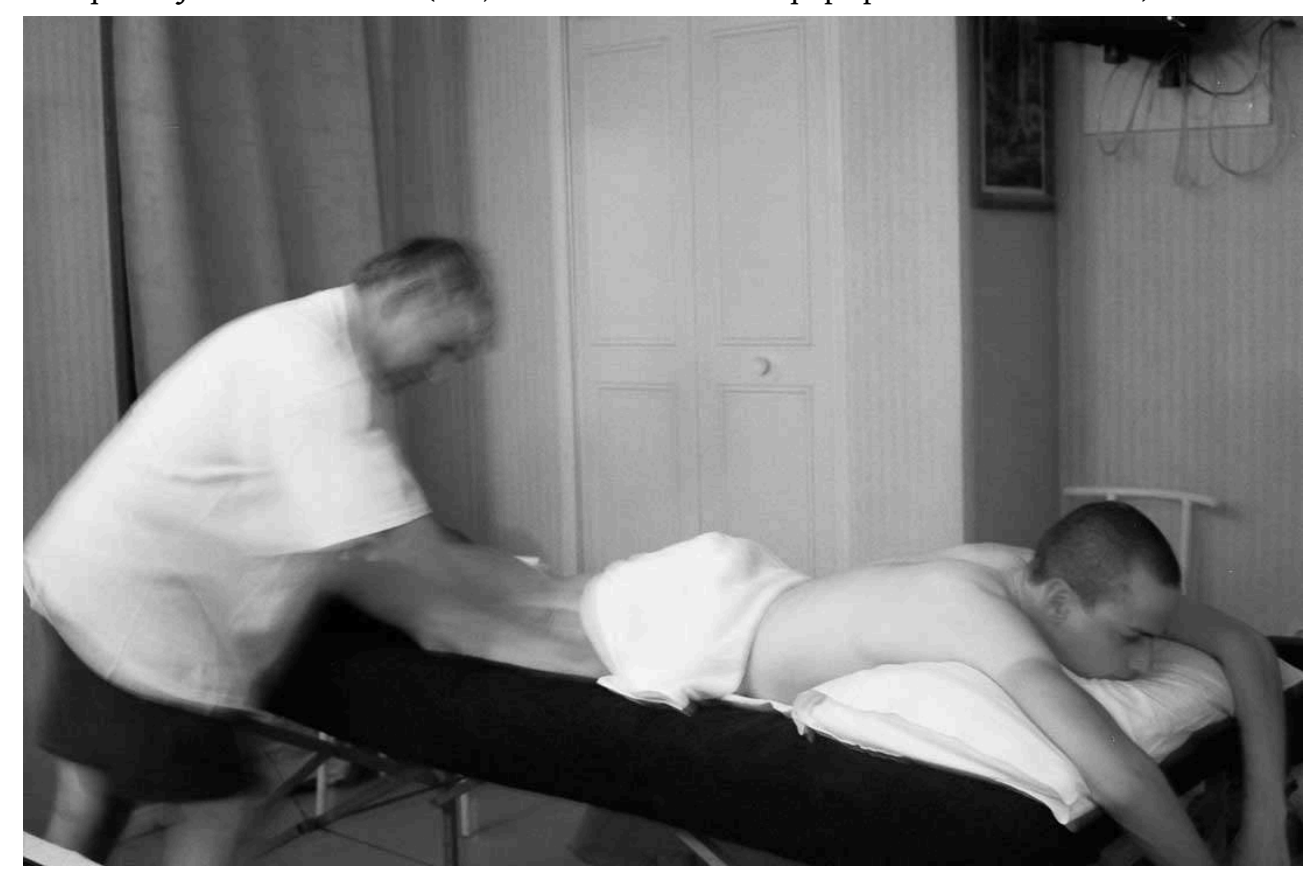

(c) X. Garnotel

Le calme et la quiétude d'une séance de massage.

La technique du massage consiste à chauffer le muscle afin de l'assouplir, de favoriser le retour veineux et lymphatique pour l'élimination des déchets liés à la contraction musculaire (notamment de l'acide lactique cristallisé dans les microfibrilles à la suite d'efforts anaérobies lactiques). Pour cela les gestes du masseur parcourent l'architecture musculaire en suivant les limites de chaque groupe musculaire en allant toujours vers le cœur (donc toujours de bas en haut pour les jambes). Mais une séance de massage ne se réduit pas à cet aspect anatomique. Chaque masseur expérimenté a 
son "toucher", ses formes de savoir corporel et technique, qui ne se verbalisent pas forcément. Les coureurs ont généralement un masseur attitré, qui les suit parfois depuis des années et auxquels ils se livrent avec confiance. Le massage a lieu chaque soir d'étape et est pratiqué depuis plus d'un siècle au sein du peloton. Il est chargé de représentations sur la façon d'évacuer le mal physique. Il s'agit d'un phénomène d'expulsion à la fois physique et symbolique que l'on peut déceler derrière le terme "sortir les toxines». Cette pratique possède une efficacité physiologique et une " efficacité symbolique " (Lévi-Strauss 1958) qui agit sur les structures organiques par un apaisement et un relâchement du tonus. Cette confiance et cette sérénité quant à l'état du corps favorisent la relaxation du tonus lors des phases préparatoires. Elles permettent également de stimuler la volonté et la motivation. Le peloton a donc construit un système de techniques du corps précis selon le cycle temporel de la saison, qui obéit à une répartition sociale des tâches. Ces techniques du corps, inscrites dans un système d'organisation sociale, donnent du sens aux actes quotidiens et permettent d'endurer l'ascèse, la rigueur et la longueur d'une saison et d'une carrière. Elles façonnent immanquablement le corps qui en devient reconnaissable et porteur de représentations propres au peloton.

\title{
Efficacité technique, esthétique fonctionnelle et style corporel
}

\begin{abstract}
«Je pense que si t'aimes pas la douleur tu fais pas de vélo. Parce que c'est un sport très dur. T'es obligé d'avoir toujours la douleur, t'as tout le temps mal aux jambes. La douleur ça fait partie du cycliste en fait, tu aimes ça ».

"Quand j'étais cyclo, j'avais pas d'objectifs, je faisais ça simplement pour mon plaisir. Quand t'as le goût de la compétition, on peut quand même réussir dans ce sport, donc on est obligé de passer par là, par la douleur. Ça te fait du bien à ton corps, ça te le sculpte. T'es obligé quoi, sinon t'es pas cycliste, ça fait partie de toi. En fait c'est du mal pour du bien » (D.C., coureur Élite 2).
\end{abstract}

\section{Transmissions et appropriations individuelles des normes techniques}

Les habiletés du coureur s'acquièrent sous différentes formes. Cela peut provenir de prescriptions, c'est-à-dire par la transmission de conseils puis de la répétition de gestes techniques normés par les entraîneurs. L'apprentissage moteur demande du temps pour être automatisé et assimilé. Même le geste apparemment simple de pédalage est soumis à un apprentissage, dès l'école de cyclisme. Cela passe en principe par des exercices d'adresse. Les coureurs en herbe réalisent pour cela des parcours balisés avec des plots où il faut slalomer, lâcher les mains, attraper un bidon au sol, accélérer et freiner, descendre du vélo en patinette et remonter en sautant directement sur la selle... Ensuite, la maîtrise du «bon coup de pédale » demande des années de pratique : choisir un braquet adapté à sa force et à ses caractéristiques musculaires, savoir " tourner les jambes sans emmener trop gros", réussir «à tirer sur les pédales pour pédaler rond». Le peloton a codifié des principes techniques dans la préparation afin de construire le "beau coup de pédale ", "rond" et fluide. L'imitation de styles faisant références à (et représentant) des modèles de pédalage ou de position est également à l'œuvre dans les apprentissages moteurs au sein du peloton. Tout comme dans la danse, « regarder, observer attentivement ou furtivement pour imiter, est une modalité 
d'apprentissage essentielle» (Faure 2000: 120). Cette modalité de transmission des techniques par l'imitation est également à l'œuvre dans le savoir-faire artisanal (Martinelli 1996).

Mais tout ne relève pas des prescriptions et de l'imitation, chaque coureur chevronné trouvant sa manière de faire en fonction de ses capacités physiques et musculaires. En effet, l'efficacité du pédalage consiste à trouver le meilleur compromis entre la vitesse et la force de pédalage. Ceci est relatif aux qualités musculaires du cycliste : certains pédalent plus en force (gros braquets utilisés) d'autres en vélocité. L'expertise technique consiste à adapter sa fréquence de pédalage à ses qualités physiques. Il existe ainsi des normes techniques transmises par les entraîneurs, mais également des adaptations spontanées selon la morphologie et les caractéristiques physiques de chaque coureur. Il existe une appropriation phénoménale, une incorporation de cette motricité atypique. Ainsi, le cycliste assimile le vélo dans son schéma corporel. Ses sensations sont liées aux paramètres du vélo: «Avoir gros " (c'est-à-dire utiliser un grand développement) procure une douleur spécifique liée à la contraction en force des muscles, « avoir petit » engendre la sensation "d'enrouler ses jambes autour de son cou », selon l'expression métaphorique du jargon. Un coureur est capable d'identifier sur son vélo les nuances des grains du bitume, la qualité du revêtement et bien évidemment la direction et la force du vent. Il y a simultanément à l'œuvre des prescriptions, des techniques du corps transmises oralement par les entraîneurs, mais aussi des adaptations et des appropriations individuelles. Le corps du cycliste, façonné et sculpté, est l'instrument malléable des normes techniques du peloton. Mais il se présente également comme l'incarnation, le moteur dynamique d'une culture technique qui évolue continuellement.

\section{L'esthétique fonctionnelle et le style corporel du peloton}

L'efficacité de ces techniques du corps façonne l'enveloppe charnelle des coureurs. Le peloton a construit des normes esthétiques basées sur la fonctionnalité. Ce qui est efficace procure une satisfaction au coureur et est jugé beau par les connaisseurs. "L'Esthétique fonctionnelle $»^{7}$ (Leroi-Gourhan 1965) du geste permet de comprendre pourquoi les sportifs peuvent passer des heures à répéter leurs gestes techniques, là où le profane ne trouve qu'ennui, pénibilité et lassitude. Ils prennent un certain plaisir dans la réalisation du bon mouvement, que ce soit « à enrouler du braquet » en cyclisme ou à trouver les "appuis» en natation ou en athlétisme. Cette répétition des gestes techniques façonne l'organisme et il est alors possible de distinguer des styles corporels propres à chaque discipline (terme qui traduit à la fois la dimension institutionnelle et comportementale).

Le style corporel ${ }^{8}$ du cycliste se caractérise par un marqueur très codifié : le rasage des jambes. Cette pratique possède une légitimité fonctionnelle puisqu'elle permet une meilleure cicatrisation en cas de chute et qu'elle favorise les séances de massage. Mais progressivement, la raison fonctionnelle est devenue anecdotique au profit de la valeur esthétique. Un coureur ayant des jambes hirsutes au départ d'une course serait immédiatement la risée du peloton. Le rasage, associé à une musculature sèche et "affûtée ", correspond à une norme esthétique et caractérise l'appartenance sociale d'un coureur. Ce rasage s'accompagne, comme nous l'avons vu, d'onctions d'huiles 
camphrées au départ des courses. Cela fait briller les jambes en faisant ressortir la musculature.

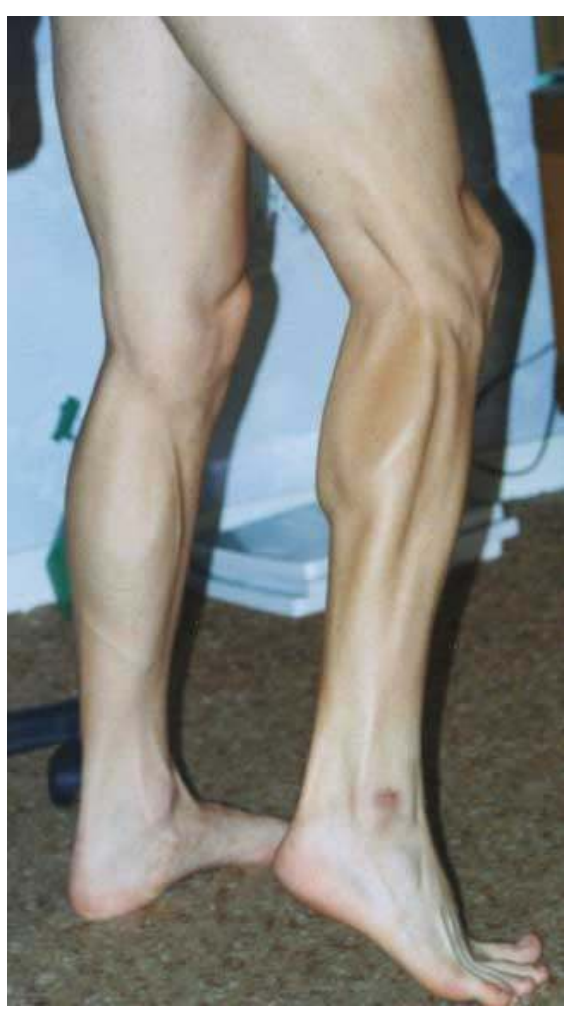

(C) X. Garnotel

Un « puncheur affûté »

Bromberger relève dans ses «trichologiques » des fonctions contradictoires concernant les traitements de la pilosité à travers les cultures si bien que l'on ne peut établir d'invariances: "d'une culture ou d'un contexte à l'autre, les mêmes signes peuvent recouvrir des significations opposées » $(2005: 12)$. Il précise toutefois que le traitement des poils renseigne constamment sur les registres du genre, des statuts sociaux et des appartenances ethniques, de l'expérience spirituelle et du sacré et enfin sur l'esthétique dominante. Le traitement de la pilosité dans le peloton renforce «la difficulté qu'il y a à élaborer une "trichologie" générale où, à chaque signe pileux, correspondrait une signification invariante » (2005: 13). En effet, le rasage des jambes est une pratique féminine au sein des sociétés occidentales, alors que la pratique cycliste féminine de compétition est très marginale et que le cyclisme renvoie indiscutablement, pour les représentations sociales, à des valeurs identifiées comme masculines telles la force, la puissance ou encore l'abnégation. Les jambes constituent le fer de lance du corps du cycliste : « avoir de bonnes jambes ", expression métonymique du peloton, signifie que le coureur est en forme. Elles traduisent, à elles seules, l'état du corps en général. Le rasage spécifique de cette partie fonctionnelle du corps du cycliste caractérise donc l'ascèse du coureur, qui met en avant les codes esthétiques du peloton. Cette technique du rasage illustre le passage d'une pratique fonctionnelle à un élément de distinction stylistique qui définit l'appartenance au milieu cycliste. Cela correspond ainsi à la notion de marqueur qui "sert à désigner, assigner et identifier autrui " (Martinelli 2005: 42). Il ne s'agit pas d'une identité ethnique qui se transmet 
nécessairement, mais d'un choix individuel de pratiquer un sport et de se définir comme cycliste (même s'il existe évidemment des influences familiales et sociales qui peuvent conditionner cet engagement avec plus ou moins de déterminismes). Ce rasage constitue ainsi le marqueur essentiel de cette appartenance, si bien que d'anciens coureurs et cyclotouristes procèdent également à cette technique corporelle.

\section{L'esthétique corporelle}

L'esthétique corporelle du cyclisme s'exprime métaphoriquement avec la notion de muscle «affûté ». Ce terme est normalement utilisé pour caractériser un couteau ou une lame aiguisée par l'action d'un artisan, afin que son outil soit plus efficace pour trancher, couper, fendre. Cette analogie traduit une esthétique de la forme rectiligne, droite, acérée, de la consistance dure et sèche à l'opposé du flasque et du mou. La volonté d'être "affûté » fait apparaître une fascination pour l'inscription de la douleur dans les tissus organiques: " T'es obligé d'avoir toujours la douleur, t'as tout le temps mal aux jambes. [...] Ça fait du bien à ton corps, ça te le sculpte » (D.C.). L'esthétique de la douleur «sculpte " le corps du cycliste. Un muscle "affutté » est esthétique pour le peloton, car il est efficace et fonctionnel : la musculature est saillante donc puissante et le tissu adipeux réduit au minimum. Il représente donc un coureur aguerri, préparé et consciencieux. Pour obtenir cet aspect, il faut nécessairement « faire le métier » que ce soit au niveau de l'entraînement ou des techniques du corps au quotidien. Parfois, chez certains coureurs, "l'affûtage » est tellement accompli qu'on le devine jusqu'aux traits du visage. Il donne une image de labeur et devient synonyme de « dur au mal».

L'esthétique corporelle du cyclisme sur route est composée d'autres éléments qui définissent ce style. Le bronzage important des bras et des jambes tranche avec la pâleur du buste. Les marques nettes de séparation de ce bronzage traduisent l'ascèse d'un coureur qui ne s'expose au soleil que pour aller rouler, à l'inverse d'un bronzage uniforme du corps qui pourrait être un signe de loisir et de vacances. On observe également une répartition asymétrique de la musculature d'un coureur qui a généralement des épaules chétives, des bras fluets, des pectoraux peu développés, un buste frêle et des jambes musclées, parfois volumineuses. Cela s'explique encore du point de vue de la fonctionnalité. Une musculature excessive des membres supérieurs est plus nuisible qu'utile pour un coureur, car cela augmente la masse et consomme de l'oxygène inutilement dans l'effort cycliste.

Martinelli définit le style de la façon suivante :

«Ensemble ou système de caractères et d'informations, qui confèrent aux choses une valeur signalétique du point de vue d'identités, de statuts, de positions sociales, le tout pour des lieux et des temps déterminés. Il est reconnaissable de manière le plus souvent contrastive, pour les membres du groupe comme pour des observateurs extérieurs. Jouant de petites ou grandes variations, il fournit un principe d'articulation double entre des normes internes et des critères externes de définition. (...) Induit par des objets, activités ou comportements, le style constitue alors le substrat d'expressions et de production, voire de modes d'existence » (Martinelli $2005: 7$ ).

33 Ainsi, l'esthétique corporelle du cyclisme ne renvoie pas aux canons esthétiques occidentaux de l'athlète qui, depuis la Grèce antique et ses sculptures, magnifient la force et la puissance à travers une musculature imposante, notamment au niveau du buste, des pectoraux et des épaules. L'esthétique corporelle du cyclisme est liée à l'esthétique fonctionnelle du geste, c'est-à-dire à l'efficacité, au rendement et à la 
performance. Les adaptations techniques vis-à-vis des propriétés de l'activité et de la tradition technique du peloton façonnent le corps. L'exigence de cette ascèse se matérialise corporellement et il est souvent possible, pour un connaisseur, de distinguer les différences de niveaux des pratiquants à partir de l'observation de leur corps. En effet, les marqueurs corporels du cycliste correspondent à des éléments de distinction stylistique permettant de définir ce que c'est que d'« être cycliste ", pour les initiés et par rapport aux autres. Le corps, ses vêtements et le matériel du coureur sont également des éléments de distinction hiérarchique au sein du peloton. La codification très précise de ce dernier permet d'identifier immédiatement le niveau des coureurs par les couleurs distinctives de leurs vélos, de leurs maillots et de leurs éventuels liserés témoignant du palmarès et de l'appartenance à une équipe prestigieuse ou non. L'allure et le style individuel renseignent enfin sur les qualités du coureur et sur ses fonctions au sein de l'équipe. Ils permettent de distinguer rapidement un leader "dégageant la classe » du simple équipier, "domestique», au potentiel banal, destiné à «porter les bidons».

$$
34 \&
$$

"Vivre cycliste " nécessite une véritable ascèse imposant des techniques du corps, normées par la culture technique du peloton. Il se dégage indiscutablement une sousculture sportive qui se caractérise par une tradition orale, une organisation sociale parfois en décalage avec les institutions réglementaires et par des codes et des traitements corporels spécifiques.

Cet ensemble d'éléments techniques et esthétiques résulte d'adaptations pragmatiques aux contraintes naturelles de l'organisme et des propriétés de l'activité. Les pratiquants ont ainsi construit des techniques corporelles et collectives afin d'être plus efficaces dans leur métier. Ces techniques façonnent l'organisme et engendrent des valeurs et des normes esthétiques. Cette codification sociale passe par des marqueurs corporels ayant une valeur stylistique qui permet de distinguer les initiés et, parmi eux, d'identifier la hiérarchie et l'organisation de cette sous-culture sportive.

\section{BIBLIOGRAPHIE}

Becker, Howard S., 1985, Outsider. Études de sociologie de la déviance. Paris, Métailié.

Brissonneau, Christophe, Aubel, Olivier \& Ohl, Fabien, 2008, L'Épreuve du dopage, sociologie du cyclisme professionnel. Paris, PUF.

Bromberger, Christian, 1986, « Hommage à André Leroi-Gourhan », Terrain n 7 : 61-76.

Bromberger, Christian, 1995, « De quoi parlent les sports », Terrain n $25: 5-12$.

Bromberger, Christian, 2005, « Trichologiques : les langages de la pilosité », in : C.Bromberger,

P. Duret, J.-C. Kaufmann \& al., Un Corps pour soi. Paris, PUF : 11-39.

Chiotti, Jérôme, 2001, De mon plein gré! Paris, Calmann-Levy. 
Cizeron, Marc, 2009, « La Croyance comme point d'appui à l'action. Étude de cas sur les croyances des enseignants ", Revue d'anthropologie des connaissances, vol. $3: 149-170$.

Clastres, Pierre, 1974, « De quoi rient les Indiens », La Société contre l'État. Paris, Éditions de Minuit.

Darbon, Sébastien, 2002, « Pour une anthropologie des pratiques sportives. Propriétés formelles et rapport au corps dans le rugby à XV », Techniques \& culture $n^{\circ} 39$. Sports et corps en jeu, novembre $2002: 1-27$.

Desgrange, Henri, 1er juillet 1903, L'Auto, quotidien sportif de 1903 à 1939.

Dodge, Pryor, 2000, La Grande histoire du vélo. Paris, Flammarion.

Faure, Sylvie, 2000, Apprendre par corps. Socio-anthropologie des techniques de danse. Paris, La Dispute.

Gaboriau, Philippe, 1995, Le Tour de France et le vélo, Histoire sociale d'une épopée moderne. Paris, L'Harmattan (« Espaces et temps du sport »).

Garnotel, Xavier, 2009a, Le Peloton cycliste, ethnologie d'une culture sportive. Paris, L'Harmattan (« Espaces et temps du sport»).

Garnotel, Xavier, 2009b, « Le Peloton cycliste : de la métaphore sociale au bouillon de culture », Revista etnografica $\mathrm{n}^{\circ} 13$, mai 2009 :17-30.

Gaumont, Philippe , 2005, Prisonnier du dopage. Paris, Grasset.

Gille, Bertrand, 1966, Histoire de la métallurgie. Paris, PUF (« Que sais-je »).

Goffman, Erving, 1973, La Mise en scène de la vie quotidienne. Paris. Éditions de Minuit.

Lagrue, Pierre, 2004, Le Tour de France, reflet de l'histoire et de la société. Paris, L'Harmattan.

Lechtman, Heather, 1977, "Style in technology - some early thoughts », in Heather Lechtman and Robert S Merrill éd., Material Culture : Styles, Organization, and Dynamics of Technology. St Paul, West pub. Co.

Lemonnier, Pierre, 1991, « De la Culture matérielle à la culture ? Ethnologie des techniques et préhistoire ", in 25 ans d'études technologiques en préhistoire. Antibes, Éditions APDCA, Juan les Pins.

Levi-Strauss, Claude, 1958, Anthropologie structurale. Paris, Plon.

Leroi-Gourhan, André, 1945, Milieu et techniques. Paris, Albin Michel.

Leroi-Gourhan, André, 1965, Le Geste et la parole - La mémoire et les rythmes, vol 2. Paris, Albin Michel.

Londres, Albert, 1924, « Les Forçats de la route », in Aimez vous les stades ? Les Origines historiques des politiques sportives en France (1870-1930), textes réunis par Alain Ehrenberg, avril 1980, Recherches numéro 43.

Martinelli, Bruno,1996, « Sous le regard de l'apprenti - Paliers de savoir et d'insertion chez les forgerons Moose du Yatenga (Burkina Faso) », Techniques \& culture ${ }^{\circ} 28$ : 9-47.

Martinelli, Brun éd., 2005, L'Interrogation du style, anthropologie, technique et esthétique. Aix-enProvence, PUP.

Mauss, Marcel, 1968, « Les Techniques du corps », Sociologie et anthropologie. Paris, PUF : 365-386. Monod, Hugues \& Flandrois, Roland, 2003, Bases physiologiques des activités physiques et sportives. Paris, Masson. 
Paturle Hervé \& Rebiere, Guillaume, 1997, Un Siècle de cyclisme. Paris, Calmann-Lévy.

Segalen Martine, 1998, Rites et rituels contemporains. Paris, Nathan.

Vigarello, Georges, 1988, Techniques d'hier... d'aujourd'hui. Paris, Laffont.

Vigarello, Georges, 1997, « Le Tour de France », Pierre Nora éd., Les Lieux de mémoire 3. Paris, Gallimard : 3801- 3833.

Voet, Willy, 1999, Massacre à la chaîne révélations sur 30 ans de tricheries. Paris, Calmann-Lévy.

\section{NOTES}

1. La méthodologie a reposé sur une observation participante de deux années (saisons 2002 et 2003) au sein du peloton cycliste amateur de série «élite 2 » (l'équivalent de la deuxième division pour les sports collectifs). Des entretiens semi-directifs ont également été réalisés avec des membres de nombreuses équipes professionnelles lors du Tour de France 2001 alors que j'étais membre de la caravane Michelin, accompagnant le responsable du partenariat de l'enseigne de pneumatique avec certaines équipes (Garnotel 2009a). Les descriptions sont accompagnées d'un grand nombre d'entretiens informels et d'expressions propres au jargon cycliste.

2. Dans leurs études sur le football et le rugby, Bromberger (1995) et Darbon (2002) ont montré «l'extraordinaire pouvoir qu'ont les propriétés formelles d'un sport donné de façonner, par elles-mêmes, des comportements et des pratiques, de les renforcer et de les constituer en système de référence [...] comme moteur d'élaboration et de consolidation d'une culture sportive » (Darbon). Ce modèle théorique met en avant les reconstructions de codes sociaux de la part des pratiquants par adaptation entre les propriétés de la pratique et les contraintes naturelles de l'espèce. Il s'inscrit ainsi plus largement dans une anthropologie des techniques humaines et interroge le processus de construction culturelle « de la culture matérielle à la culture?» (Lemonnier 1991). Cette perspective anthropologique illustre le dynamisme des créations culturelles par les adaptations techniques du pragmatisme humain : de la préhistoire et de l'impact des industries lithiques sur l'organisation sociale des communautés de chasseurscueilleurs aux sous-cultures contemporaines comme il en est question dans cet article.

3. Le calendrier des compétitions cyclistes est organisé de façon traditionnelle et respecte un ordre précis. On retrouve chaque année les mêmes compétitions aux mêmes périodes. Elles sont derechef rassemblées en groupes d'épreuves caractéristiques, comme les classique flandriennes (Paris-Roubaix, le Tour des Flandres) du mois de mars, exigeant force et robustesse; les classiques ardennaises (Flèche Wallonne, Liège-Bastogne-Liège), propices aux "puncheurs ", puis les courses par étapes pour les coureurs complets. Enfin, les critériums estivaux sont des courses en circuits, généralement organisées autour d'évènements festifs. Ils permettent au public de voir les coureurs qui se sont illustrés sur des courses médiatisées. Ils n'ont pas la même valeur en terme de palmarès mais présentent un intérêt financier pour les coureurs grâce aux primes et aux prix des courses.

4. Sur le plan historique, l'éthique amateuriste coubertinienne reposait sur la volonté aristocratique de maintenir une distinction sociale en se faisant garante d'une mentalité sportive «noble et désintéressée. Mais le sport cycliste s'est démarqué dès la fin du xixe siècle par l'émergence d'une forte dynamique professionnelle sous l'impulsion des célèbres marques de cycles qui parrainaient les grandes épreuves à des fins de notoriété. Si bien que l'amateurisme a rapidement été substitué par une culture populaire plus pragmatique. En effet, en favorisant des systèmes de récompenses généreux, l'Union vélocipédique de France, créée en 1890, a incité les coureurs des classes populaires à participer aux épreuves et à remporter ces gains. Par exemple, le Grand Prix de l'Union Vélocipédique de France offrait 10000 francs au vainqueur, alors qu'à 
titre indicatif, le salaire annuel d'un instituteur était d'environ de 1000 francs (Lagrue P. 2004). Ce système de récompense et cette appropriation populaire de la pratique ne sont sans doute pas étrangers à la régulation spontanée du cyclisme sur route. En effet, le sport cycliste se caractérise sociologiquement par l'origine populaire de ses pratiquants et également par une faible emprise institutionnelle des fédérations, si on le compare à d'autres sports comme l'athlétisme (Brissonneau, Aubel \& Ohl 2008). Les récentes affaires de dopage, depuis 1998, marquent tout de même un tournant dans les pratiques des coureurs et les politiques institutionnelles. Il faut noter une baisse évidente des pratiques amateurs de "bricolage", liée à une lutte anti-dopage drastique de la F.F.C. (Fédération française de cyclisme). Il est très difficile de connaître les substances du dopage au plus haut niveau mondial puisque les générations de biotechnologies évoluent rapidement, et ce, dans la plupart des grands sports spectacles. Certains biotechnologues évoquent l'utilisation à venir de thérapies génétiques permettant d'augmenter les capacités motrices, les champions d'aujourd'hui préfigurant alors peut-être l'humain de demain...

5. Le statut d'un coureur de l'élite passe principalement par ses fonctions au sein de son équipe. La hiérarchie d'une équipe s'effectue à partir du potentiel de chaque individu, d'où l'adage «le cyclisme est un sport individuel qui se pratique en équipe ". Il s'agit de soumettre les plus forts ou les plus adaptés à un type d'épreuve dans les meilleures conditions pour s'imposer. Ainsi, avant une compétition, chaque équipe possède généralement un "leader " déterminé. Les "équipiers » lui apportent de l'eau, l'aident à "remonter le peloton» et l'accompagnent en cas d'incident mécanique ou de chute. Il faut noter que le statut de leader peut évoluer au cours de la saison. En fonction des caractéristiques physiques d'une épreuve (plate, vallonnée ou montagneuse), ainsi que des aptitudes physiques des membres de l'équipe et de leur état physique du moment, le directeur sportif détermine un leader qui dispose des privilèges de ce statut. Le corps témoigne de ces qualités respectives. Tels des animaux racés, les spécialistes d'une de ces compétences cyclistes se repèrent immédiatement. Le "routier-sprinter" est généralement puissant et musculeux, le «puncheur » est tonique et dynamique, alors que le « grimpeur » est en principe fin et élancé.

6. Il faut noter une évolution quant aux fonctions et au statut de coach. À la «belle époque ", les aristocrates ou les bourgeois encadraient souvent leurs «poulains». En effet, ils trouvaient un certain prestige dans la gestion des sportifs «professionnels». Sur le modèle équestre, ces notables possédaient leurs «bêtes de courses" (Vigarello 1997) et ils s'occupaient de leur préparation. Progressivement, avec la popularisation de ce sport et son appropriation par les pratiquants, d'anciens coureurs charismatiques sont devenus à leur tour des directeurs sportifs comme ce fut le cas de Geminiani pour Anquetil ou encore de Guimard pour Hinault. L'organisation s'est complexifiée à partir des années 80 et de l'apparition des sciences du sport et de l'augmentation des budgets des équipes sponsorisées au sein de la société de spectacle et de consommation moderne. Il faut alors noter une division et une spécialisation des tâches avec un « coach », qui est le directeur sportif ou le chef, et des mécaniciens, des masseurs, des médecins et des préparateurs physiques (pour l'entraînement, la diététique...).

7. Même si Leroi-Gourhan s'inscrit dans une perspective évolutionniste et que l'anthropologie a réalisé des découvertes depuis les écrits de cet auteur, ce concept "d'esthétique fonctionnelle » doit être conservé comme un élément central, permettant de comprendre le lien existant entre l'efficacité technique et la satisfaction, le plaisir esthétique ressenti par l'artisan, l'artiste ou le sportif lors d'une réalisation matérielle ou technique.

8. Lechtman utilise le terme de "style technologique» pour caractériser des « dispositifs matériels solidifiés par un modelage culturel latent » (1977:4- 7). 


\section{RÉSUMÉS}

Le Peloton cycliste.De la culture technique à la sous-culture sportive. Les structures techniques de la bicyclette ainsi que le contexte socio-économique des sociétés industrielles ont façonné les propriétés du cyclisme sur route. Les pratiquants se sont adaptés pragmatiquement aux contraintes physiques de ce sport en construisant des organisations collectives, des techniques corporelles et des codes sociaux. Ainsi, l'objectif de cet article est d'étudier comment ces éléments techniques se sont cristallisés en marqueurs stylistiques intégrant une esthétique corporelle et révélant des codes hiérarchiques propres au peloton.

The Cyclist Bunch. From technical culture to sports subculture. The technical structures of the bicycle as well as the economic and social context of the industrial societies have shaped the properties of road cycling. The followers adapted themselves to the physical constraints of this sport by constructing collective organizations, body techniques and social codes. Thus, the object of this article consists in studying how these technical elements crystallized in stylistic codes integrating a corporeal aesthetic and revealing hierarchical codes typical of the bunch.

INDEX

Keywords : aesthtics, anthropology, body, sports, style, technical

Mots-clés : anthropologie, corps, esthétique, sports, style, techniques

\section{AUTEUR}

XAVIER GARNOTEL

IDEMEC UMR 6591

xavier.garnotel@orange.fr 